\title{
Multi-User Augmented Reality with Communication Efficient and Spatially Consistent Virtual Objects
}

\author{
Xukan Ran \\ Computer Science \& Engineering \\ University of California, Riverside \\ Riverside, CA, USA \\ xran001@ucr.edu \\ Kittipat Apicharttrisorn \\ Computer Science \& Engineering \\ University of California, Riverside \\ Riverside, CA, USA \\ kapic001@ucr.edu
}

\author{
Carter Slocum \\ Computer Science \& Engineering \\ University of California, Riverside \\ Riverside, CA, USA \\ csloc001@ucr.edu \\ Maria Gorlatova \\ Electrical \& Computer Engineering \\ Duke University \\ Durham, NC, USA \\ maria.gorlatova@duke.edu
}

\author{
Yi-Zhen Tsai \\ Computer Science \& Engineering \\ University of California, Riverside \\ Riverside, CA, USA \\ ytsai036@ucr.edu \\ Jiasi Chen \\ Computer Science \& Engineering \\ University of California, Riverside \\ Riverside, CA, USA \\ jiasi@cs.ucr.edu
}

\begin{abstract}
Multi-user augmented reality (AR), where multiple co-located users view a common set of virtual objects, is becoming increasingly popular. For example, Google Just a Line allows multiple users to draw virtual graffiti in the same physical space. Multi-user $\mathrm{AR}$ requires network communications in order to coordinate the positions of the virtual objects on each user's display, yet there is currently little understanding of how such apps communicate. In this work, we address this key gap in knowledge by showing that the communicated data directly impacts the latency and positioning of the virtual objects rendered on the users' displays. We develop solutions to these problems that we find along three facets: (1) efficient communication strategies that trade off communication latency for spatial consistency of the virtual objects; (2) a new metric that enables mobile AR devices to update their virtual objects as they move around and observe more of the scene; and (3) a tool to automatically quantify how much the virtual objects' positions inadvertently change in time and space. Our evaluation is performed on Android smartphones running open-source AR. The results show that our system, SPAR, can decrease the latency by up to $55 \%$, while decreasing the spatial inconsistency by up to $60 \%$, compared to baseline methods.
\end{abstract}

\section{CCS Concepts}

- Human-centered computing $\rightarrow$ Ubiquitous and mobile computing; • Networks; • Information systems $\rightarrow$ Multimedia information systems;

\section{Keywords}

Augmented reality, multi-user AR, latency, spatial inconsistency

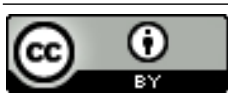

This work is licensed under a Creative Commons Attribution International 4.0 License.

CoNEXT '20, December 1-4, 2020, Barcelona, Spain

(C) 2020 Copyright held by the owner/author(s).

ACM ISBN 978-1-4503-7948-9/20/12.

https://doi.org/10.1145/3386367.3431312
ACM Reference Format:

Xukan Ran, Carter Slocum, Yi-Zhen Tsai, Kittipat Apicharttrisorn, Maria Gorlatova, and Jiasi Chen. 2020. Multi-User Augmented Reality with Communication Efficient and Spatially Consistent Virtual Objects. In The 16th International Conference on emerging Networking EXperiments and Technologies (CoNEXT '20), December 1-4, 2020, Barcelona, Spain. ACM, New York, NY, USA, 13 pages. https://doi.org/10.1145/3386367.3431312

\section{Introduction}

Augmented Reality (AR) applications have recently exploded in popularity among smartphone users. In AR, a user's field-ofview (FoV) is overlaid with virtual objects, which should remain fixed with respect to the real world in order to provide a seamless transition between the real world and the virtual objects. As AR becomes more popular, a natural question is: can we share the virtual objects with other users? Based on the off-the-shelf AR apps currently available, the answer is "yes". For example, Pokemon Go released the Buddy Adventures feature in December 2019, which allows multiple users to view their virtual creatures together in the same real world space. Other multi-user AR applications currently available include Just a Line, where multiple users can collaboratively draw virtual graffiti, and Minecraft, where users can build structures together from virtual blocks.

Yet despite their emerging popularity, little is known about the network communications of multi-user AR apps. The fact that these apps involve multiple users clearly indicates that some form of network communication is required. However, it is currently unknown how these apps communicate, what they are communicating, and how the data communications impact user experience. This work seeks to address this key gap in knowledge, and propose solutions to the problems that we find in this space.

Through our measurements of off-the-shelf multi-user AR apps (detailed in Sec. 2), we find that users experience multiple seconds of latency between one user placing a virtual object to it appearing on another user's display. Moreover, the virtual objects can appear at different locations, with respect to the real world, on each user's display. These two problems - latency and spatial inconsistency - are key factors in AR user experience [13, 29, 51], and we find that they depend on the communicated information between the AR devices. Thus the goal of this work is to optimize the network 
communications of multi-user AR apps, to enable fast and accurate coordination of the virtual objects across AR displays. For example, in a classroom, students equipped with AR devices should be able to see the same virtual chemistry molecule and manipulate it, with the virtual molecule remaining consistent across students. However, uncoordinated or laggy updates to the virtual molecule would break the illusion of seamless integration with the real world, and result in artifacts such as other users appearing to touch non-existent parts of the virtual molecule.

We meet and address several technical challenges towards realizing this vision. (1) Firstly, multi-user AR apps share a large initial data burst containing information about the real world environment, in order to render the virtual objects at the correct locations on each AR display. This leads to multiple seconds of latency whenever users move to a new area and wait for virtual objects to appear. To address this, we propose communication strategies that adapt to the positions of the virtual objects in order to reduce communication latency. (2) Next, as the user moves around, the AR device continuously observes new information about the real world. These observations can be processed to update the virtual objects' positions with respect to the real world, but the update may actually harm the positioning accuracy if the wrong information is used. To address this, we propose a new "feature geo distance" metric that allows the AR app to select the right camera frames to re-align the virtual objects' positions with other users. (3) Finally, multi-user AR apps do not know if their virtual objects are drifting in time or in space. Without knowing that the virtual objects are experiencing positioning issues, these apps does not know when problems need to be corrected. To address this, we develop a methodology and tool to automatically quantify spatial inconsistency issues of the virtual object across time and across users.

While there has been research on object detection and cloud/edge offloading for $\mathrm{AR}$ (e.g., [27, 32, 42]), these works typically consider a single user viewing the virtual objects, rather than multi-user coordination. They also do not incorporate simultaneous localization and mapping (SLAM), which is part of off-the-shelf AR systems today to enable 3D understanding, but contribute to the latency and spatial inconsistency issues that we observe. Industry players have started to look at multi-user AR [8, 20, 35], but focus mainly on application development and not the communication aspects of the underlying platform. To the best of our knowledge, no prior work has systematically examined AR spatial consistency issues when there are multiple users, and their dependency on the network data transmissions between the devices.

In summary, we study the emerging application of multi-user $\mathrm{AR}$ and its networking aspects. In particular, we focus on rendering virtual objects that are clustered around a common "anchor" point in the real world [20]; this is common in typical multi-user AR apps (e.g., all the virtual Pokemon or virtual graffiti are placed near each other). We call our system SPAR (SPatially Consistent AR). Our contributions include:

- We measure the performance and network usage of off-the-shelf AR apps, and identify problems of high latency, spatial drift, and spatial inconsistency of the virtual objects over time and across users.

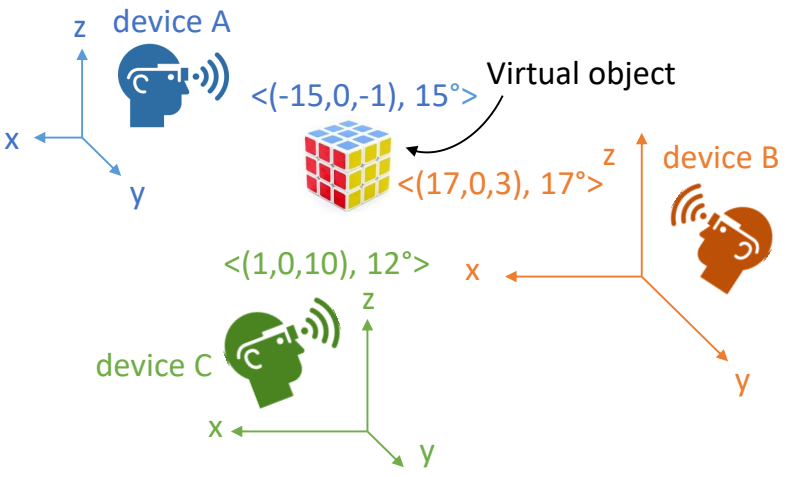

Figure 1: Multiple AR devices try to ensure consistent views of a virtual object, despite different coordinate systems.

- We develop new methods for efficient communication and computation of the AR devices. Specifically, SPAR performs the following: (i) it adapts the communicated information to the virtual object positions, to reduce latency; (ii) it efficiently computes the virtual objects' positions in each user's display through lightweight coordinate system alignment, to create spatial consistency; (iii) it continuously updates the positions of the virtual objects with respect to the real world using a new update metric, to maintain spatial consistency; and (iv) it automatically quantifies the spatial drift and consistency of the virtual objects, to evaluate performance.

- We perform evaluation on Android AR devices, extending VINS [31], a 6 DoF-based AR platform, with multi-user capabilities. We work with open-source systems because existing AR platforms from Android, Apple, and Microsoft [8, 20,35] are closed source and thus their internal code cannot be modified for experimentation. Our results show that SPAR can decrease the total latency by up to $55 \%$, while decreasing the spatial inconsistency of the virtual objects by up to $60 \%$, compared to a baseline method of communicating the full data or off-the-shelf AR platforms. We also show that our tool can estimate a virtual object's spatial drift and inconsistency with a low RMSE of only $0.92 \mathrm{~cm}$, compared to manual human labeling.

In the remainder of this paper, we discuss the measurements that motivate this work (Sec. 2), a brief background (Sec. 3), the overall system architecture (Sec. 4), and the design of the individual components (Sec. 5). We then evaluate the system (Sec. 7), discuss related work (Sec. 9), then conclude (Sec. 10).

\section{Motivation: Spatial Drift, Inconsistency, and Latency of Off-the-Shelf Mobile AR}

To showcase the issues of virtual object spatial inconsistency and latency in multi-user AR, we examined several off-the-shelf AR Android apps. The four Android apps we tried were Google CloudAnchor [20], Google Just a Line [21], Minecraft [37], and Pokemon Go [39], running on Pixel 4 smartphones. We have two users, Alice and Bob, who perform the following sequence of user interactions:

(1) Place initial virtual object: Alice places a virtual object by tapping the screen. 

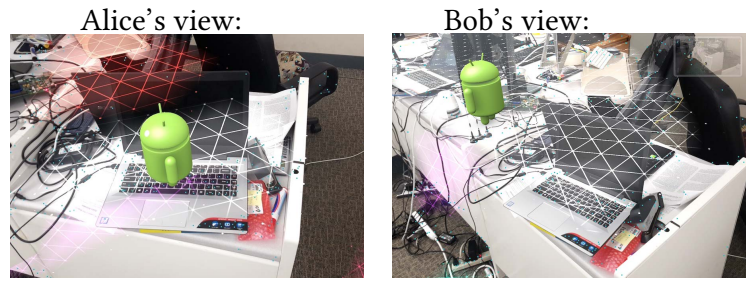

(a) Spatial inconsistency across two AR devices.

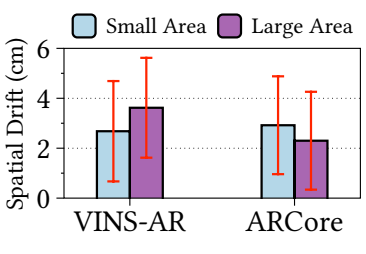

(b) Spatial drift

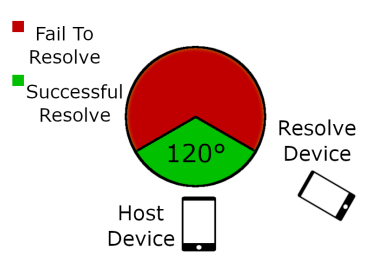

(c) Spatial inconsistency

Figure 2: Quantifying spatial drift and inconsistency. The resolving devices suffer from spatial drift of 2-4 cm and fail to resolve if their orientation with respect to the host is more than $60^{\circ}$.

(2) Render initial virtual object: Bob waits for it to appear on his screen.

(3) Subsequent user interactions: Alice taps the screen to move a virtual object or place new virtual objects (only Just a Line and Minecraft have this feature). Bob waits for the update to be reflected on his screen.

Our main finding is that there is significant delay and spatial inconsistency between when Alice places the first virtual object and Bob renders it on the screen (steps 1 and 2 above), thus motivating the work in this paper. Similar results hold when there are multiple receiving users (i.e., multiple Bobs) who wish to view Alice's virtual objects. Below, we detail our quantitative measurements.

Spatial drift and inconsistency: We experimented 5 times with CloudAnchor, with each trial lasting 1 minute with Bob moving $1 \mathrm{~m}$ (small area) to $4 \mathrm{~m}$ (large area) in the real world. In these experiments, we observed two types of spatial issues relating to the virtual object's position:

- Spatial drift: For a single user, the virtual object can drift in position over time.

- Spatial inconsistency: When there are multiple users, the virtual object can appear in a different location, relative to the real world, on each user's display, as shown in Fig. 2a.

In the single-user case (Fig. $2 \mathrm{~b}$ ), the spatial drift is around $2-3 \mathrm{~cm}$ in both the large and small areas, for both ARCore [19] and VINSAR [31] platforms. In the multi-user case, spatial inconsistency results are larger (results later in Sec. 7), and moreover we observe that when the two devices are placed more than $60^{\circ}$ apart, the virtual object fails to resolve, as shown in Fig. 2c. We also observed similar issues with Magic Leap, but focus on smartphone-based AR in this work due to the ubiquity of smartphone devices. These spatial drift, inconsistency, or failure to resolve a virtual object can cause serious issues when multiple users interact in a shared AR session (e.g., multiple users jointly building a tower of building blocks), and are key contributors to user experience in AR [29, 51]. This motivates SPAR's goal of reducing spatial drift and inconsistency. Similar to prior work [47], we focus on positioning errors, as we did not observe much rotational error our experiments. The low rotational error we observe may be due to our use of visual-inertial SLAM as the basis for our AR system, which generally has less rotational error than pure visual SLAM [28].

Additionally, from running these experiments, we found that manually measuring the position of the virtual object is laborious and time-consuming, with each measurement requiring several seconds per frame for a human to examine the image and record

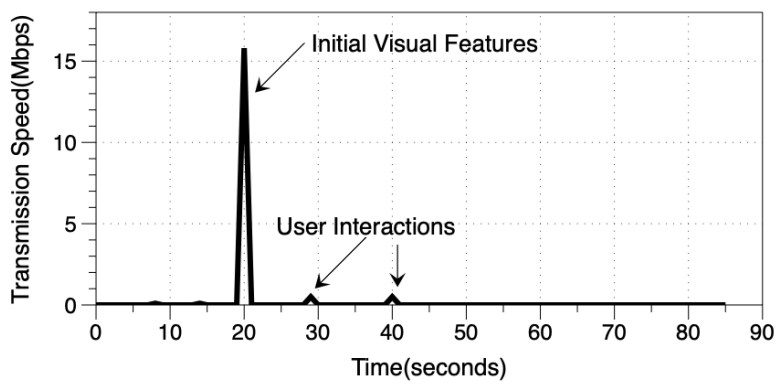

(a) Just a Line network trace.

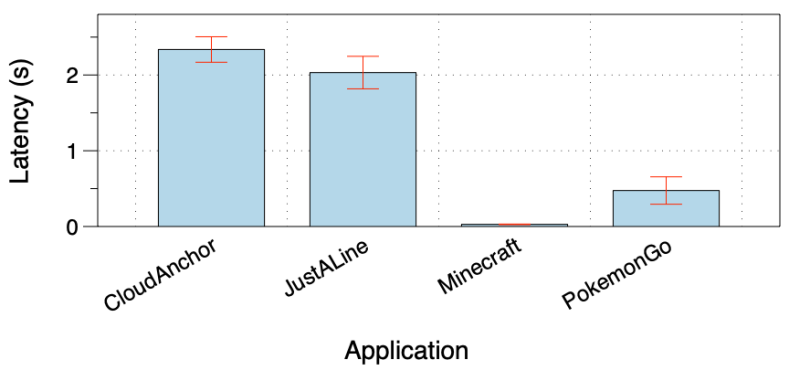

(b) Communication latency.

Figure 3: AR apps transmit a large amount of environment data for the initial virtual object, resulting in mult-second communication latency.

the virtual object's position. This motivates SPAR's spatial drift and inconsistency estimation tool, to reduce the amount of human labor and enable AR apps to automatically quantify these issues.

Communication latency: We next examine the data transfer between the AR users. Fig. 3 shows a representative network trace from Just a Line (other apps have a similar pattern, not shown for brevity). We observe a large data transfers for the initial object placement (steps 1 and 2), 9-20 Mb, and smaller data transfers during the subsequent user interactions (step 3), <100 kb. These large initial data transfers prolong the communication latency. With an upload bandwidth of $8 \mathrm{Mbps}$ (the average in our lab), the initial communication latency is plotted in Fig. 3b. The communication latency of $>2 \mathrm{~s}$ for the ARCore-based apps (CloudAnchor and Just a Line) is a lower bound on the user-experienced delay (while Minecraft and Pokemon Go have lower communication latency, they suffer from higher spatial inconsistency). This latency is a 
key contributor to user experience in multi-user AR [13,51], thus motivating SPAR's goal of reducing user-perceived latency.

Connection between latency and spatial inconsistency: We discover there is a critical connection between the aforementioned spatial inconsistency and latency issues we observed. Off-the-shelf AR platforms use similar methods $[8,20,35]$ to share real world environment information between users during step 1, resulting in the large spikes of data observed in Fig. 3. This environmental information helps align the coordinate systems of the AR devices, enabling each device to render the virtual objects at fixed positions in the real world. In other words, the data communicated during the placement of the initial virtual object (step 1) directly impacts the spatial drift and inconsistency. This motivates SPAR's communication-efficient strategies to decrease communication latency, while trading off with spatial drift and inconsistency.

\section{Brief Background on AR}

Current AR platforms such as Google ARCore, Apple ARKit, and Microsoft Hololens rely on simultaneous localization and mapping (SLAM). SLAM solves the problem of when a device is in an unknown environment, how to build a consistent map and localize itself at the same time [15]. In a typical single-user AR scenario, the AR device uses SLAM to construct a point cloud representing the $3 \mathrm{D}$ coordinates of features in the real world, and also estimates its own location and orientation (known as pose). To compute the point cloud, SLAM selects a subset of camera frames (known as keyframes), extracts features from the keyframes, runs SLAM algorithms on the features, and outputs the 3D coordinates of the features in each keyframe (i.e., the point cloud) and the estimated device pose. These SLAM algorithms includes keyframe matching to localize the device with respect to its past trajectory. Each keyframe data structure contains feature descriptors, 2D feature coordinates with respect to the camera image, and 3D feature coordinates with respect to the real world. The $3 \mathrm{D}$ feature coordinates are relative to an origin point in the real world, which is called the device's world coordinate system.

To render virtual objects for AR, the device records the pose of the virtual object (defined as its location and orientation, which can be provided by user input or by an object detector). The AR device runs SLAM continuously to update its own pose estimate and the 3D coordinates of the features in the point cloud, and then draws the virtual object on the display when its FoV overlaps with the virtual object's pose.

\section{System Architecture}

SPAR consists of the following components that work together in concert, as shown in Fig. 4. For illustrative purposes, we describe a hosting device (Alice, the "host") who places the virtual objects, and a resolving device (Bob, the "resolver") who wishes to view the same virtual objects. When there are multiple resolvers (i.e., multiple Bobs), each Bob performs steps 2 and 3 below.

(1) Adaptive AR communications: A naive approach to share AR information between Alice and Bob would be sending all of Alice's SLAM data to Bob(s). However, this approach is not cognizant of AR, and can be very slow; To reduce this communication latency, we design an intelligent mechanism that adapts the data transmissions to the content of the AR scene. Specifically, only areas of the real

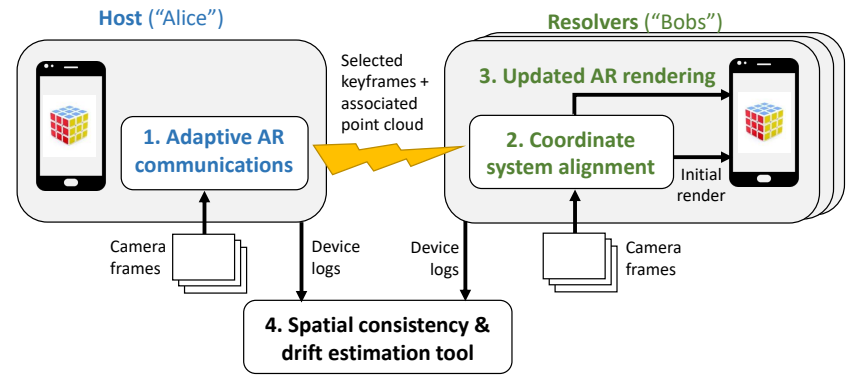

Figure 4: SPAR system overview. The modules work together for fast multi-user AR with spatially consistent virtual objects.

world in which Alice was near the virtual objects, and the virtual objects were visible, are sent to Bob(s).

(2) Coordinate system alignment: Using the received information, Bob next desires to render the virtual objects at the correct locations in the real world. However, since Bob and Alice can open the AR app from different positions in the real world, they lack a common frame of reference to accurately describe the pose (location and orientation) of the virtual objects. Current single-user $\mathrm{AR}$ does not have this problem because there is only one device and thus one world coordinate system. We propose a lightweight coordinate system alignment method, which allows Bob to align himself inside Alice's coordinate system. The key idea is to reuse some functionality from single-user AR for path loop detection [41], which that is already running on the device, making our method very lightweight yet accurate.

(3) Updated AR rendering: After the initial rendering of the virtual objects, Bob may continue to move around the world. As Bob observes new information about his environment, he can use this information to re-align his coordinate system with Alice and update the positions of virtual objects. But how can Bob know if these updated positions are more or less accurate? To enable Bob to predict whether the spatial inconsistency improved with the new observations, we propose a new metric, feature geo distance, which has good correlation with spatial inconsistency. The intuition behind this metric is that if Bob observes areas close to the virtual objects, these observations can likely improve spatial consistency.

(4) Spatial inconsistency and drift estimation tool: The above operations can affect where the virtual object is rendered on Bob's display. We develop a spatial inconsistency and drift estimation tool to quantify these impacts on the rendered virtual objects. This tool runs as an overlay in SPAR, collecting logs from the AR devices, and computing the drift and spatial inconsistency offline. The chief challenge lies in finding a fixed real-world reference point from which to accurate measure how the position of the virtual object changes over time or across users.

\section{System Design}

In this section, we discuss the main modules of SPAR: adaptive AR communications (Sec. 5.1), coordinate system alignment (Sec. 5.2), updated AR rendering (Sec. 5.3), and the spatial inconsistency and drift estimation tool (Sec. 5.4). 


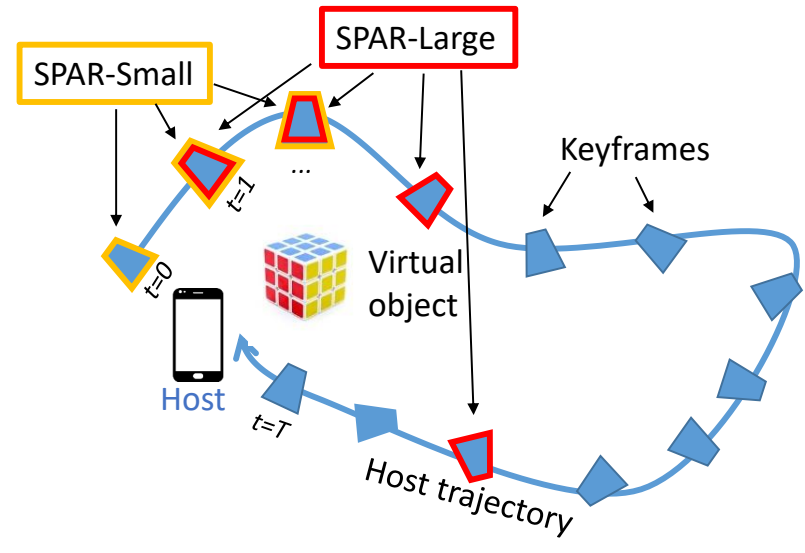

Figure 5: Adaptive AR communication strategies. SPAR-Small selects keyframes and point clouds from when the host creates a virtual object. SPAR-Large selects information from when the host is near a virtual object and it is visible.

\subsection{Adaptive AR Communications (Host)}

To align the positions of the virtual objects with respect to the real world in each AR display, the host sends a set of keyframes, the associated point cloud with those keyframes, and the virtual objects' coordinates to a resolver. A virtual object's coordinates are a small $4 \times 4$ transformation matrix, but set of keyframes can become very large if many keyframes are sent. While a simple strategy is to send all of the keyframes, this can grow very large as the hosting device continues to collect more data, incurring significant communication latency. For example, in our experiments (details in Sec. 7), the host collects approximately 110 keyframes after 1-2 minutes, consuming $40 \mathrm{Mb}$ even after applying standard compression techniques [1], resulting in 5 seconds of communication latency on an $8 \mathrm{Mbps}$ uplink connection. Note that these 110 keyframes were already downsampled by SLAM; in other words, the basic downsampling done by SLAM is insufficient and still results in high communication latency. We therefore ask: Can we adapt which keyframes and associated point cloud information are sent, in order to decrease the communication latency?

Our idea is to intelligently adapt what information is sent based on the AR scene, balancing between sending enough information to enable good coordinate system alignment between the host and resolver, but less information to reduce the communication latency. We base our strategies on an insight unique to the AR setting: only keyframes close to the virtual object in time and space are needed. We propose two adaptation strategies: (1) an aggressive SPAR-Small strategy (yellow outline in Fig. 5), which only selects the keyframes that are close in time to when the virtual object was created, and (2) a more comprehensive SPAR-Large strategy (red outline in Fig. 5), which only selects keyframes from when the host is near the virtual object, and the virtual object is visible on the display.

The intuition behind the SPAR-Small strategy is that when the host creates the virtual object by tapping on the device's display, the information about the scene observed around this time is likely sufficient for a resolver to recreate the same virtual objects. The intuition behind the SPAR-Large strategy is two-fold: visibility is important because if the virtual object is not visible on the screen,

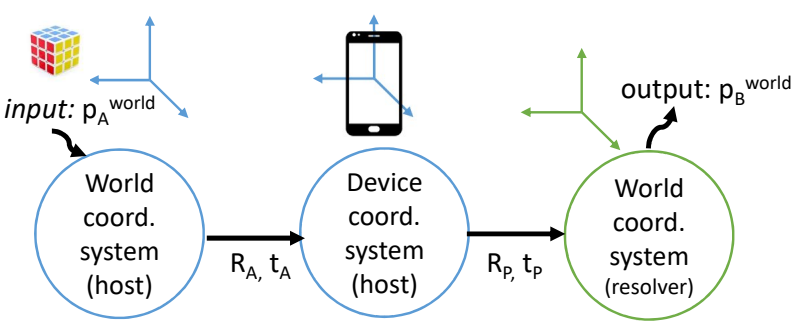

(a) Overall process.

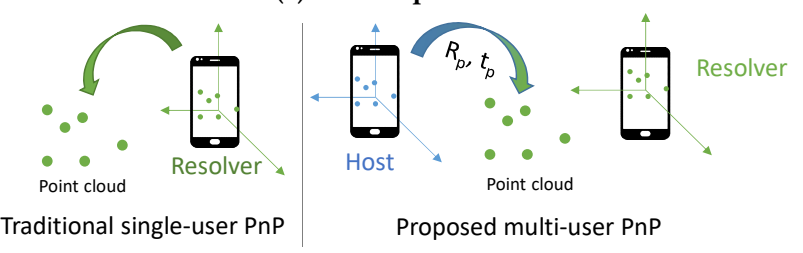

(b) Multi-user PnP to compute $R_{p}, t_{p}$

Figure 6: Coordinate system alignment to transform a virtual object from the host's world coordinate system to the resolver's world coordinate system.

the camera is usually facing another direction and the observed features may not be useful; and nearness is important because if the host moves in a large environment, the number of keyframes may be large, so nearness can filter out far-away keyframes. The output of this module is a reduced set of keyframes and their associated point clouds, $F_{A}$, sent from the host to a resolver.

\subsection{Coordinate System Alignment (Resolver)}

Next, given that a resolver has received the set of keyframes and associated point cloud $F_{A}$ and the virtual object's coordinates $p_{A}^{\text {world }}$ in the host's world coordinate system, how can a resolver determine the position of the virtual objects in its own world coordinate system, which is needed for rendering? The main challenge is that each device's world coordinate system is initialized at an arbitrary origin point (typically wherever the device is when the AR application is launched), and the devices may be launched randomly by users from different locations in space. Some existing AR apps [21,39] side step this problem by requiring that the users open their AR apps when the phones are exactly side-by-side, but this is an unnecessary burden on user experience. The goal of the SPAR's coordinate system alignment module is to enable the AR apps to launch from arbitrary starting points, while correctly positioning the virtual objects in each user's AR display.

Multi-user PnP: The specific technical goal is to transform a virtual object's coordinates, which are provided in the host's world cooordinate system (left circle in Fig. 6a) to a resolver's world coordinate system (right circle in Fig. 6a). An intermediate step is the host's device coordinate system (middle circle in Fig. 6a). Since the host knows the definitions of its own world coordinate system and its device coordinate system (left to middle circle in Fig. 6a), it can estimate the appropriate transformation $R_{A}, t_{A}$ (i.e., its pose) using SLAM (line 5 in Alg. 1). However, the missing transformation is from the host's device coordinate system to a resolver's world coordinate system, $R_{p}, t_{p}$ (middle to right circle in Fig. 6a). 
Our key idea is to use real-world features, which remain fixed, to estimate this transformation. Specifically, we propose a multiuser PnP method, which is a re-purposing of the single-user PnP method [52] that already runs on AR platforms. We utilize existing functions in a new way in order to avoid implementation of complex new functionality and keep the system lightweight. However, the existing single-user PnP function can only query for a given keyframe within a device's own world coordinate system, not between two devices' coordinate systems. For example, when running on Bob's device, the inputs to single-user PnP are a query keyframe with associated point cloud, and the output is the an estimate of Bob's pose in Bob's world coordinate system (left side of Fig. 6b). However, for multi-user PnP, we realize that we can query one of Alice's keyframes instead, and compute the pose of Alice's device in Bob's coordinate system (right side of Fig. 6b), using exactly the same $\mathrm{PnP}$ function. This gives us the transformation $R_{p}, t_{p}$ (line 6 in Alg. 1), and enables us to compute the complete chain of transformations $R_{p}\left(R_{A} p_{A}^{\text {world }}+t_{A}\right)+t_{p}$ (line 7 in Alg. 1) needed to render the virtual objects in Bob's display.

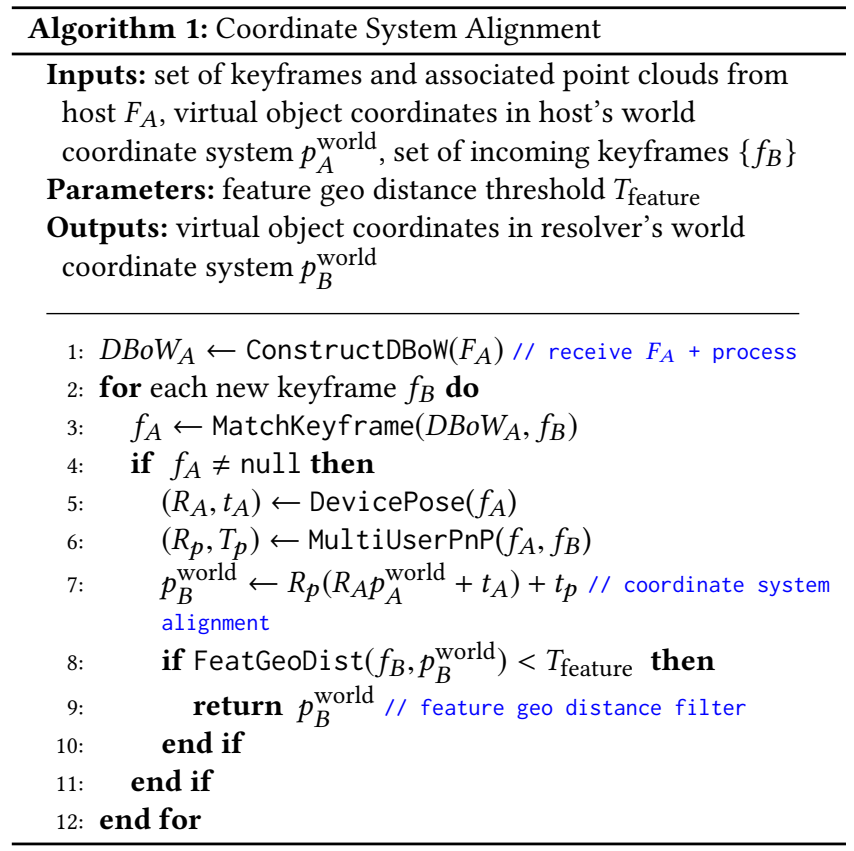

\subsection{Updated AR Rendering (Resolver)}

Given the initial rendered virtual objects from Sec. 5.2, we next discuss what happens as a resolver moves around and collects more information about the real world. Can this new information be used to re-compute the coordinate system alignment and update the poses of the virtual objects for improved spatial consistency? A naive solution of using the most recent keyframe and associated point cloud to update the poses of the virtual objects can lead to bad results, however, if the keyframe was observed at a distant point far from the virtual object. This is because the coordinate systems would be well aligned at that distant point, but lose alignment in areas closer to the virtual objects, resulting in spatial inconsistency with other users. So when should the new information be used?

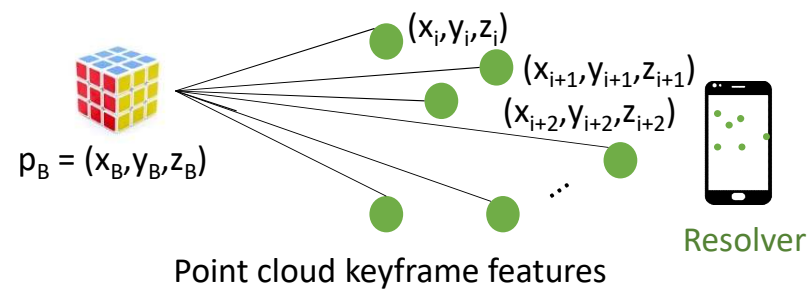

Figure 7: Feature geo distance is the average distance from real world features to a virtual object.

Our approach is to develop a new metric to help estimate which new keyframes and associated point clouds will produce the best alignment and improve the spatial consistency. Simple estimation schemes such as selecting keyframes from the host and resolver with the lowest pixel-by-pixel differences or Hamming distance are possible, but in our experience, this results in keyframe matches that are distant from the virtual object, resulting in poor coordinate system alignment and thus spatial inconsistency issues.

Instead, we leverage our understanding of the AR scenario: features near the virtual object are more important for coordinate system alignment. We propose a new metric based on this, "feature geo distance". The feature geo distance is defined as the average distance to a virtual object over the common features in a pair of matched keyframes, as illustrated in Fig. 7. Specifically, given a set of features $i$ with coordinates $\left(x_{i}, y_{i}, z_{i}\right)$ from keyframe $f_{B}$, and the $3 \mathrm{D}$ coordinates of the virtual object $p_{B}^{\text {world }}$, we compute the feature geo distance as:

$$
\text { FeatGeoDist }\left(f_{B}, p_{B}^{\text {world }}\right)=\frac{\sum_{i \in f_{B}}\left\|\left(x_{i}, y_{i}, z_{i}\right)-p_{B}^{\text {world }}\right\|}{M}
$$

where $M$ is the number of common features. We compute the feature geo distance on each pair of matched frames, and only update the pose of a virtual object if the feature geo distance is less than a threshold (line 8 in Alg. 1). Essentially, we use feature geo distance as a predictor of spatial inconsistency (this correlation is evaluated in Sec. 7.3).

\subsection{Spatial Inconsistency and Drift Estimation Tool}

The last module of SPAR seeks to quantify the impact of the above modules on spatial inconsistency and drift. This is contrast to typical AR error estimation, where human eyes can be used, which can be inefficient [23]; Manual labeling by human eyes is time consuming if the virtual object's position needs to be labeled every frame, and also be inaccurate, if there are no real-world distance measures visible in the frame (e.g., a meterstick). In this section, we discuss our design of a tool to automate and improve human eyeball-based accuracy estimation.

Overall idea: Our method, in short, is to place one or more markers (e.g., ArUco markers [44]) into the real world before running the AR app. The markers' location and orientation can be accurately estimated by the devices using $\mathrm{PnP}[2,52]$, and used as reference points from which to measure and compare the virtual object's position as rendered by each device. We use ArUco markers as opposed to natural features in the scene because pose 


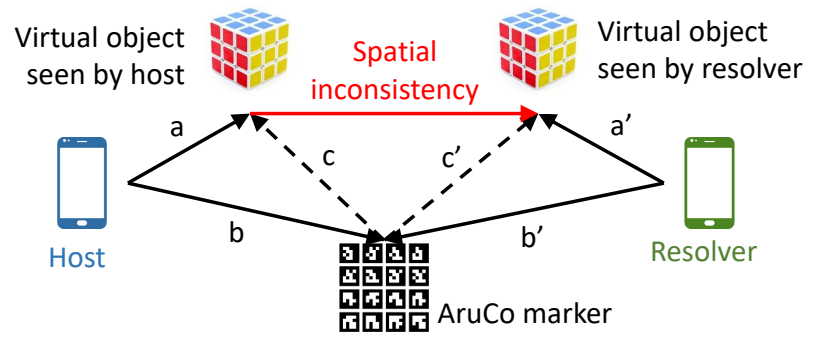

Figure 8: Conceptual example of computing spatial drift and inconsistency.

estimation from natural features is not accurate enough for our purposes, resulting in extremely noisy tool output.

As shown in Fig. 8, Alice and Bob will draw the virtual object at $a$ and $a^{\prime}$, respectively, relative to their current pose. In an ideal multi-user AR scenario, the virtual object's resulting locations (end of $a$ and $a^{\prime}$ arrows) are exactly the same; however, in practice they can be spatially inconsistent (red line in Fig. 8). In order to measure the difference between the endpoints of $a$ and $a^{\prime}$ (i.e., the spatial inconsistency), the marker is used as a common reference point. Specifically, we develop the following methodology:

(1) Using the keyframe $f$ and $f^{\prime}$ from the host and a resolver, respectively, the tool calculates the $b$ and $b^{\prime}$ vectors from the device to the marker (see Fig.8) using the marker present in the keyframe and the single-user PnP method. (lines 1-2 in Alg. 2)

(2) The $c$ and $c^{\prime}$ vectors from the real-world marker to the virtual anchor are computed as $c=a-b, c^{\prime}=a^{\prime}-b^{\prime}$. (lines 3-4 in Alg. 2).

(3) The tool outputs $\left\|c-c^{\prime}\right\|$ (length of the red line in Fig. 8), which is the magnitude of the spatial inconsistency between the two devices. (line 5 in Alg. 2).

The above procedure estimates the spatial inconsistency of multiple users at a single instance in time. A similar procedure can be used to compute the spatial drift of a single user over time, by letting $a, b, c$ be Alice's observations at $t=0$, and $a^{\prime}, b^{\prime}, c^{\prime}$ be Alice's observations at $t=1$. The output $\left\|c-c^{\prime}\right\|$ in that case represents the spatial drift between $t=0$ and $t=1$. An additional wrinkle is that the above steps rely on $a, b, c$ being in the same coordinate system. However, because they are computed by different functions in the AR platform, they typically are not output in the same coordinate system, because of the way that the real world and the devices are modeled and stored in AR/VR systems [30, 45]. We use a variation of the techniques described in Sec. 5.2 to reconcile these coordinate systems and produce the spatial inconsistency and drift estimates.

\section{Implementation}

AR devices: We use Samsung Galaxy S7 smartphones running Android 8 as the AR devices. Our AR system is developed in C++ with the Android NDK on top of VINS [31, 36, 41], an open-source AR platform, which we extend with multi-user capabilities with 3000 additional lines of code. VINS has comparable baseline spatial drift issues as ARCore, as shown in Fig. 2b, so we believe it is a reasonable platform on which to test our proposed systems. We

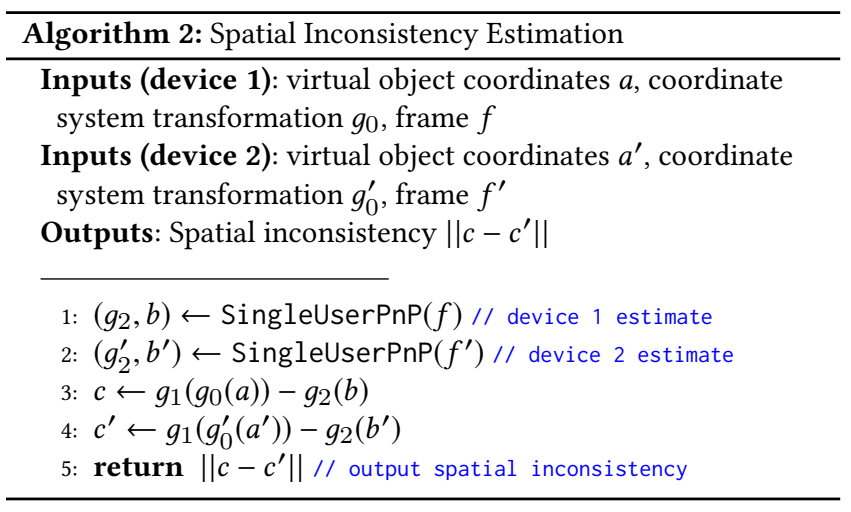

use FAST feature and BRIEF descriptors. For coordinate system alignment, we use DBoW2 [17] to speed up keyframe matching, OpenCV [9] for basic PnP functionality, and Boost [1] for data serialization and compression for efficient network transmissions. Communications are accomplished through sockets, where the host acts as a socket server and the resolvers connect to download the set of keyframes via a TCP connection. The devices are connected to a TP-Link AC1900 WiFi router.

Replay framework: To enable experimentation with different algorithms under repeatable conditions (e.g., the same mobility patterns of the users), we developed a replay framework that replays the same sequence of camera frames from the host and a resolver, and allows SPAR or other baselines to be run on top. This involves saving the host's point cloud, keyframes, and virtual objects' coordinates, along with the resolvers' point clouds and keyframes. Then for each trial, the framework loads the host's point cloud and keyframes, run the desired algorithms, and emulates a resolver's experience as new camera frames arrive one-by-one, adaptive communications occur, and coordinate system alignment and updated $\mathrm{AR}$ rendering are performed. If successful, the virtual object will be drawn on the resolver's keyframes and we record the latency and spatial inconsistency.

Tool: The spatial drift and inconsistency tool runs offline on an edge server in our implementation; in general, it could be run on any device, including one of the AR devices themselves. It is written in Python3 using OpenCV [10], Numpy, and its quaternion library. The tool takes as input the keyframes from each AR device, the AR app's log of the corresponding virtual object positions and orientations, the measurements of the ArUco marker, and the calibration matrices of AR device cameras. Since that different AR platforms (e.g., VINS, ARCore) typically output data in slightly different formats, we wrote ad hoc parsing and conversion functions for VINS.

\section{Evaluation}

We evaluate SPAR's performance in terms of computation and communication latency, bandwidth consumption and spatial inconsistency. The main findings are that SPAR-Small and SPAR-Large lower average latency by up to $55 \%$ on average, and provide improved spatial inconsistency, especially when a virtual object first appears, by $11 \%-60 \%$, compared to baseline approaches. 


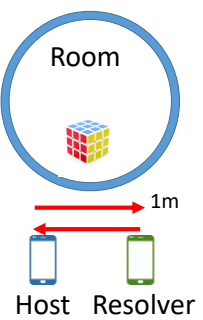

Scenario 1

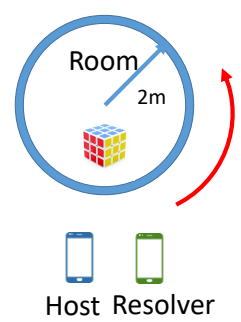

Scenario 2

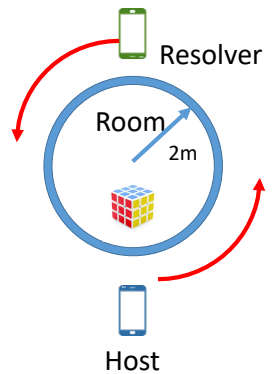

Scenario 3

Figure 9: User mobility pattern test cases.

\subsection{Setup}

Scenarios: We perform experiments in the lab and in a home environment. A host places a virtual object (in our case, a virtual cube), walks around the area, and sends the relevant data once to a resolver. This resolver then walks around the scene and tries to render the virtual cube at the correct location. We evaluate the performance from the point of view of a resolver at two time instances: (i) initially, when the virtual object is first displayed (Sec. 7.2), and (ii) subsequently as the resolver continues to move around and update the virtual object's position (Sec. 7.3). We also evaluate the performance of the tool to estimate spatial drift and inconsistency during these two phases (Sec. 7.4). The average WiFi speed was 8 Mbps upload and $50 \mathrm{Mbps}$ download in the lab, and $8 \mathrm{Mbps}$ upload and $20 \mathrm{Mbps}$ download at home.

The user mobility pattern test cases are illustrated in Fig. 9 and described below.

- Scenario 1: Small area: The host and a resolver are mostly stationary, and move within a $1 \mathrm{~m} \times 1 \mathrm{~m}$ area.

- Scenario 2: Large area + same initial position: The host and a resolver start at the same place facing the same direction. Each user moves independently within a $4 \mathrm{~m} \times 4 \mathrm{~m}$ area.

- Scenario 3: Large area + different initial position: The host and resolver start at different places. They both move independently within a $4 \mathrm{~m} \times 4 \mathrm{~m}$ area.

Each scenario is repeated 25 times, with each trial lasting $40-90$ seconds.

Baselines: Along with the SPAR-Large and SPAR-Small strategies proposed in Sec. 5.1, we compare against several baselines, All and ARCore:

- All: The host sends all keyframes and associated point clouds (already downsampled by SLAM) to a resolver.

- SPAR-Small: The host sends 5 keyframes and their associated point clouds from before and after creating a virtual object (10 time instances total). This strategy geared towards small environments.

- SPAR-Large: The host sends keyframes and associated point clouds for which the virtual object is visible within the FoV and the host is within $T_{\text {keyframe }}=3 \mathrm{~m}$ of the virtual object. This strategy is more conservative and geared towards large environments.

- ARCore [19]: ARCore is a highly optimized, closed-source production level AR platform with cloud processing. We include

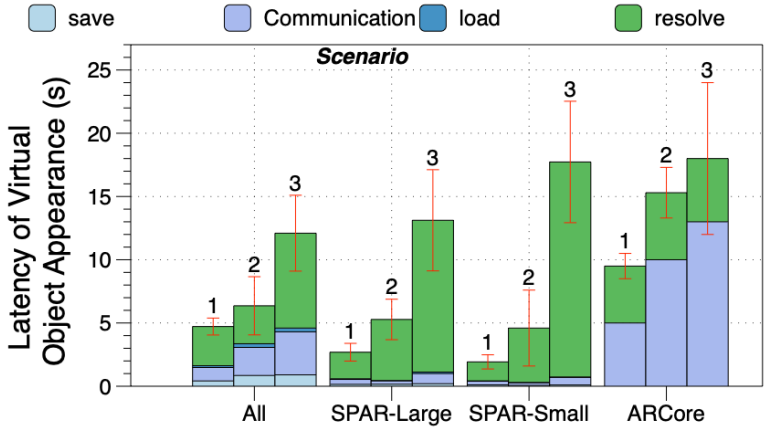

Adaptive AR communication strategies

Figure 10: SPAR reduces total latency by up to $55 \%$ compared to All and ARCore baselines, on average. Note that the latency here is the initialization latency, when the user first loads the AR app. Once this initialization has happened, subsequent updates to the virtual objects' locations and orientations happen in real-time.

this comparison for reference; our goal is to showcase the improvements of our proposed methods in the open-source VINS platform, which could then be incorporated into optimized production platforms. Due to API restrictions, the ARCore experiments differ slightly in that VINS creates a virtual object before the host moves, while ARCore creates it after the host moves.

We also experimented with two other baselines using the individual nearness and visibility criteria from SPAR-Large, but their results were similar to the other baselines and omitted. We didn't compare performance with other AR platforms such as Hololens or ARKit because they run on different hardware (Hololens, iPhone/iPad), so it is difficult to have a fair comparison with SPAR, which is prototyped on Android (although its methods are generalizable). Metrics: We evaluate several metrics:

- Latency of Initial Virtual Object Appearance (s): This latency consists of several components:

- Save: The time the host spends to adapt the AR data in preparation for transmission.

- Communication: The time spent to communicate the selected data to a resolver. For ARCore, this includes the cloud processing time.

- Load: The time a resolver spends to load the host's data and initialize SLAM processing.

- Resolve: The time for a resolver to move close to a virtual object and perform coordinate system alignment.

- Spatial drift and inconsistency (cm): As discussed in Sec. 2, spatial drift is defined as the distance that a resolver's virtual object changes over time(assuming a ground truth stationary virtual object). Spatial inconsistency is defined as the distance between a host and resolver's virtual object instances at a given time.

- Failure rate: A virtual object can fail to appear on a resolver's screen if the coordinate system alignment cannot find similar 


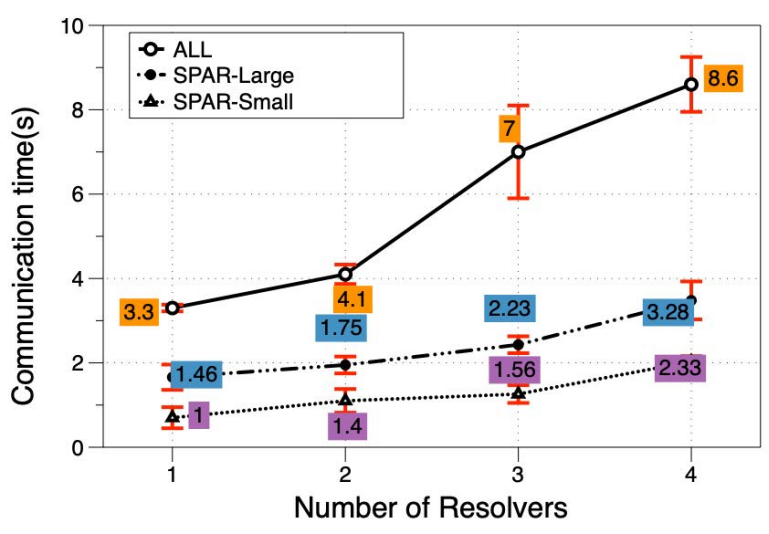

Figure 11: SPAR scales communication time with the number of resolvers.

enough matching frames. We count the number of times this failure occurs.

\subsection{Initial AR rendering}

We first discuss the initial rendering of the virtual object on the resolving user's display. We seek to answer the following questions: Does the adaptive AR communication strategy reduce latency? Are the virtual objects rendered with low spatial inconsistency?

Latency: We plot the average latency of a virtual object's initial appearance, along with its breakdown, in Fig. 10 for each scenario and baseline method. The SPAR-Large and SPAR-Small strategies generally have lower latencies than the All and ARCore baselines, with SPAR-Small performing well in small environments like scenario 1, and SPAR-Large generally performing well in larger environments such as scenarios 2 and 3 . The All baseline generally has higher latency than SPAR because it sends the full AR data, resulting in more than 3 seconds of communication latency. One exception is scenario 3, where SPAR takes more time than All. This is because the latency measurement includes the time for the user to walk closer to the virtual object. SPAR uploads fewer keyframes and typically needs more time to find a keyframe match in scenario 3, but once the virtual object does appear, it has significantly lower spatial inconsistency, as discussed later on in Fig. 12a. This illustrates the tradeoff between latency and spatial inconsistency. We also note that scenario 3 is considered a challenging scenario, with many off-the-shelf AR apps (such as Pokemon Go and Just a Line) simply avoiding such scenarios by asking players to stand side-by-side during initialization.

Finally, the ARCore baseline also has high total latency, because it sends large amounts of data for cloud processing. In general, there is a tradeoff between the communication and resolve latency: a low communication latency (as in SPAR-Small) implies scanty information for coordinate system alignment, so a resolver has to take more time to find a match before coordinate system alignment is successful, resulting in higher resolve latency.

In summary, the SPAR-Large strategy achieves good balance of communication and resolve latency, and can save an average of $15 \%$ total latency compared to All and $40 \%$ compared to ARCore, on average across all scenarios.

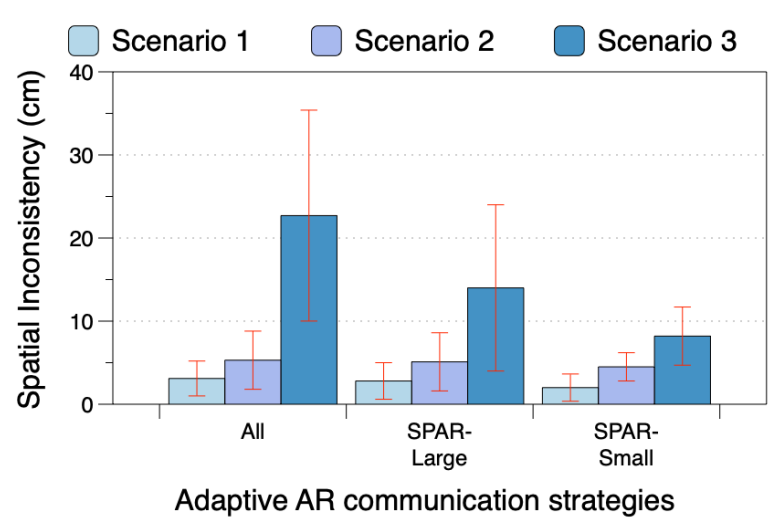

(a) First appearance of virtual object.

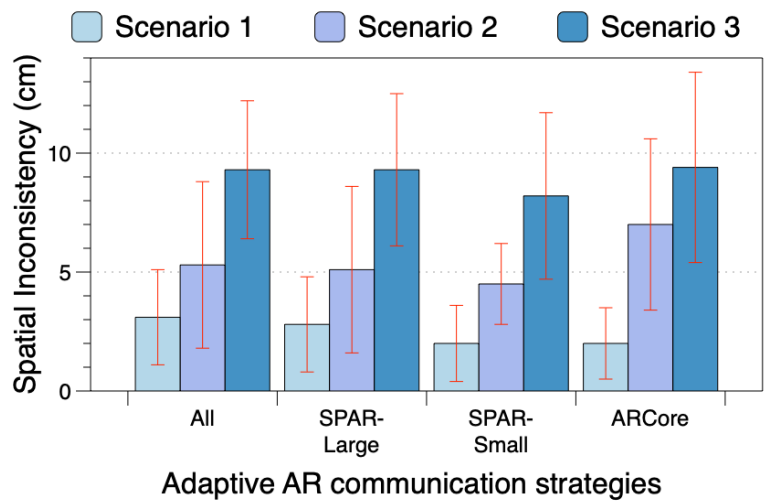

(b) Resolver $1 \mathrm{~m}$ away from virtual object.

Figure 12: SPAR improves spatial inconsistency, especially in large areas (scenarios 2,3 ) by $11 \%-60 \%$ on average, compared to All and ARCore.

Scalability: We also examine how SPAR scales as the number of resolvers increases, by varying the number of resolvers from 1 to 4 . Since all of the resolvers communicate with the host simultaneously over a shared bottleneck wireless connection, we focus on the communication latency only, as the save, load and resolve processes run in parallel on the individual devices and thus scale up easily. The average communication latency across all scenarios is shown in Fig. 11. Both SPAR-Small and SPAR-Large scale well with the number of resolvers, with approximately $0.5 \mathrm{~s}$ of latency for each additional resolver. However, All suffers from long communication latency when there are more than 2 resolvers.

Spatial inconsistency: We next examine the virtual objects' spatial inconsistencies, and plot their mean and standard deviation at two time instances: when a virtual object initially appears on a resolver's display, typically far away (Fig. 12a), and later when a resolver moves closer, around $1 \mathrm{~m}$ from a virtual object (Fig. 12b). The reason we plot two different time instances is because as a resolver moves closer to a virtual object, it observes more information about the environment and can update the position of the virtual object, changing the spatial inconsistency values.

SPAR-Small performs well at the initial appearance of the virtual object ( $<8 \mathrm{~cm}$ spatial inconsistency in all scenarios), and reduces 
the spatial inconsistency as the resolver gets closer to the virtual object. One drawback of SPAR-Small is that it has high latency in the large environments it was not designed for (see Fig. 10). In large environments (scenarios 2 and 3), SPAR-Large has lower spatial inconsistency than the All baseline when the virtual object first appears (Fig. 12a), and compared to the ARCore baseline when close to a virtual object (Fig. 12b). Hence SPAR-Small and SPAR-Large work well for the respective environments they were designed for. Examples from scenario 2 are shown in Fig. 13.

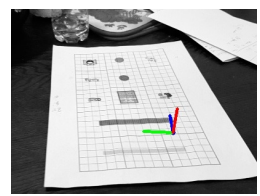

(a) Host (b) SPAR-Small

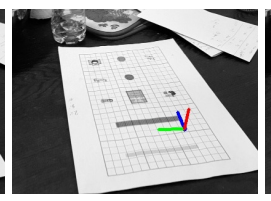

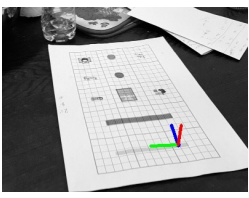

(c) SPAR-Large
Figure 13: Screenshots of the virtual object seen by the resolver under different adaptive AR communication strategies.

Surprisingly, the All baseline does not have the lowest spatial inconsistency, despite communicating full information about the environment. This is because the abundance of information sometimes results in coordinate system alignment far from the virtual object, leading to poor alignment near the virtual object and thus spatial inconsistencies. ARCore performs worse in the larger scenarios 2 and 3 when a resolver is close to the virtual object (Fig. 12b). Note that we do not record ARCore's initial spatial inconsistency because the resolver is too far away from the virtual object to measure clearly (SPAR does not have this issue because it can produce detailed logs for analysis).

In summary, SPAR-Small's spatial inconsistency in small scenarios ranges from $2-3 \mathrm{~cm}$ at a virtual object's first appearance, which is $20 \%$ better than the All baseline; while SPAR-Large achieves 6-9 $\mathrm{cm}$ spatial inconsistency in large scenarios, which is $11 \%-35 \%$ better than ARCore when near a virtual object. SPAR's accuracy in scenario 1 and 2 is generally consistent with or improves over ARCore, with most challenging scenario being scenario 3, where SPAR still outperforms ARCore on average.

Failure rates: In our experiments, SPAR-Small failed to resolve twice in scenario 2 . Since we have 75 trials total across scenarios, this gives a failure rate of $2.7 \%$. The cause of failure may be because SPAR-Small too aggressively reduces the amount of AR data transmitted, as it only save 10 keyframes and their associated point cloud, making it hard to perform coordinate system alignment and render a virtual object. The other baselines did not fail throughout our experiments, so on the whole, despite SPAR-Small having lower spatial inconsistency and good latency, SPAR-Large is preferable in general for its more consistent performance.

\subsection{Subsequent AR rendering}

In this section, we isolate the impact of SPAR's "Updated AR Rendering" module (Sec. 5.3). To validate our hypothesis that feature geo distance correlates with spatial inconsistency (see Sec. 5.3), we plot the spatial inconsistency versus feature geo distance in Fig. 14a over 3 trials. Each point on Fig. 14a represents a specific pair of matched keyframes; the y-axis records the spatial inconsistency resulting from coordinate system alignment with that matched pair. We can see that as the feature geo distance increases, spatial inconsistency gets worse. This suggests that feature geo distance can be used to select good keyframes for coordinate system alignment, and thus improves the virtual object's spatial inconsistency.

Since we use a feature geo distance threshold $T_{\text {feature }}=3 \mathrm{~m}$ in Alg. 1, in Fig. 14b we plot the average spatial inconsistency and standard deviation when the feature geo distance is less than and greater than the threshold. It includes 6 trials and 310 matched keyframes, with 170 frames having geo distance less than $3 \mathrm{~m}$, and 140 frames greater than $3 \mathrm{~m}$. The average spatial inconsistency for frames with feature geo distance greater than $3 \mathrm{~m}$ is nearly 40 $\mathrm{cm}$, but applying the threshold filters out those frames and reduces spatial inconsistency by more than $50 \%$. This reinforces our message that the feature geo distance can be an efficient way to filter out keyframe matches that result in larger spatial inconsistency.

Finally, to illustrate how the feature geo distance metric impacts $\mathrm{AR}$ rendering, in Fig. 14c we plot the time series of a particular trial in scenario 2. We compared our "feature geo distance filter" approach (blue line) to a simple "no filter" baseline (red line) that updates a virtual object's position using the resolver's most recent keyframe. Since in scenario 2, a resolver is initially near the virtual object, then moves away, then moves close again, the expectation is that the feature geo distance of the most recent keyframe will follow a similar pattern, first being low, then high, then low, Because the baseline approach uses the most recent keyframe for matching, this suggests that the virtual object's spatial drift will get worse and then better. Fig. 14c shows the baseline approach matches our expectation, while our proposed approach achieves a better (lower) spatial drift by intelligently selecting the right keyframes according to the feature geo distance metric.

In summary, the feature geo distance metric provides a good way to select which keyframe the resolver should use to update the virtual object's position, and can reduce spatial drift by $50 \%$ on average compared to a baseline "no filter" approach of using the most recent keyframe for coordinate system alignment.

\subsection{Spatial Drift and Inconsistency Tool}

In this section, we evaluate the final component of SPAR, the spatial drift and inconsistency tool proposed in Sec. 5.4. We wish to compare the $\mathrm{drift} /$ inconsistency values reported by the tool vs. the human-observed values, in order to evaluate the tool's accuracy. We first evaluate the tool's performance qualitatively. We plot an example time series of the tool's output in Fig. 15b. This time series shows that the virtual object moves by less than $3 \mathrm{~cm}$ every 1 second or so, which we qualitatively observe to be true during the experiment. To understand these results, in Fig. $15 \mathrm{c}$ we plot the trajectory of the resolver in space, with respect to the virtual object (blue line, $a$ from Fig. 8) and ArUco marker (red line, $b$ from Fig. 8). These trajectories are identical, except for an offset, as expected since they are with respect to different reference points. However, it is when this offset changes over time $(c=a-b)$ that spatial drift occurs. We can see this in Fig. 15b and Fig. 15d, where the circled points correspond to varying offset and thus higher spatial drift.

To evaluate the tool's performance quantitatively, we prepare the following test setup. We place a real $1 \mathrm{~cm} \times 1 \mathrm{~cm}$ grid paper 


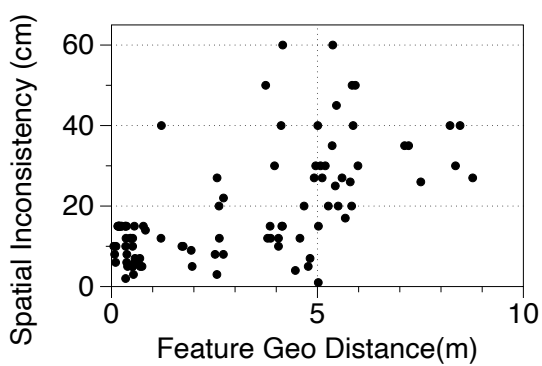

(a) Correlation.

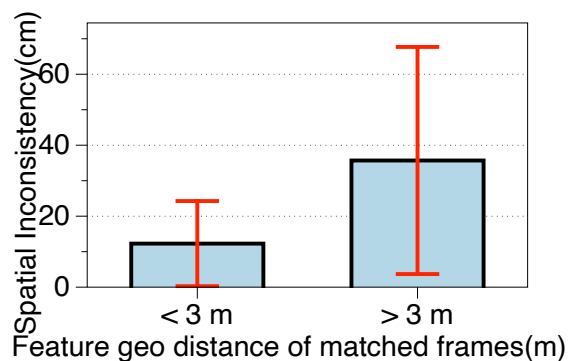

(b) $T_{\text {feature }}$ threshold.

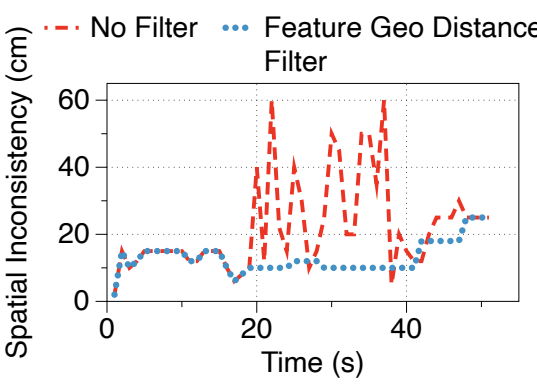

(c) Time series.

Figure 14: The feature geo distance metric filters good keyframes for coordinate system alignment, resulting in lower spatial inconsistency for a resolver.

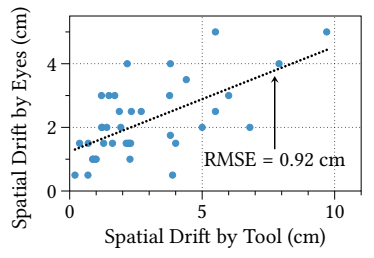

(a) Manual vs. automatic labeling.

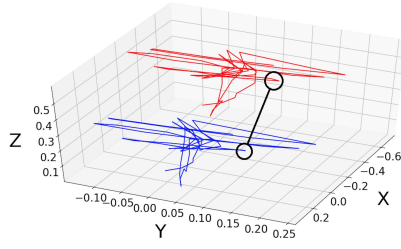

(c) Trajectory of the device.

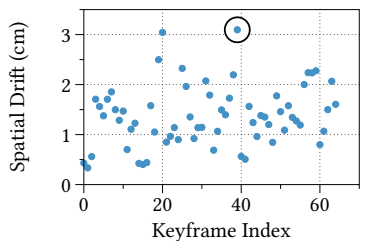

(b) Spatial drift over time.

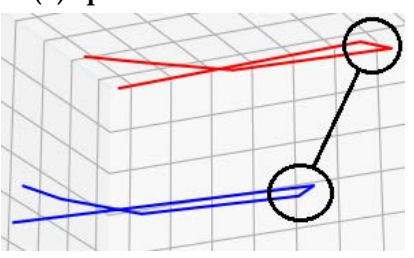

(d) Zoomed in of (c).

Figure 15: Spatial drift and inconsistency estimation tool. The tool matches manual human labeling with an RMSE of $0.92 \mathrm{~cm}$.

in the scene, initialize the virtual object on top of the grid paper, and painstakingly go through each keyframe and manually record the coordinates of the virtual object on the grid paper. We then choose random pairs of keyframes and plot the spatial drift from the manual labeling vs. the spatial drift output by the tool. Fig. 15a shows the results. The RMSE is $0.92 \mathrm{~cm}$. We see good agreement between the manual labels and the tool's output, indicating that our proposed method can successfully estimate spatial drift (spatial inconsistency is computed in a similar manner). Any disagreement between the manual labels and the tool's output are, we believe, due to fundamental limitations of SLAM in computing the device trajectory (e.g., Fig. 15c), which the tool relies on. In terms of computation time, the tool is able to generate estimates for tens of keyframe pairs in about one second, whereas manual labeling by humans takes several seconds per keyframe pair.

\section{Discussion}

Multiple virtual objects: Although our experiments focused on sharing one virtual object between users, SPAR could easily generalize to multiple virtual objects, because the common coordinate system it computes $(\S 5.2)$ can be used to represent the poses of multiple virtual objects. Specifically, for each resolver, coordinate system alignment would be performed once, and each virtual object projected and rendered onto the AR display based on its pose in the computed coordinate system. This would result in the each virtual object experiencing similar spatial inconsistency and latency as the single object case.

Scalability: The spatial inconsistency experienced by SPAR users would not be substantially impacted as number of users increases. This is because each resolver performs its computations ( $§ 5.2, \S 5.3)$ in parallel with other clients, so the computed coordinate system, virtual object poses, and hence spatial inaccuracy results of each resolver are independent of each other. This is similar to performing a 2-user (a host and a resolver) experiment multiple times. The main performance bottleneck that depends on the number of users is the communication bandwidth, which impacts the latency, as shown in Fig. 11 and discussed in $§ 7.2$.

SLAM and marker-based AR: SPAR is designed for SLAMbased AR, which is common in off-the-shelf AR systems such as Google ARCore, Apple ARKit, and Microsoft Hololens. We use VINS-MONO [31] as the basis for our AR system, which is designed for static environments, so SPAR inherits these limitations (SLAM in dynamic environments is an active area of research). Another class of AR systems is marker-based AR [18]. Since marker-based AR only need the marker information to position and render the virtual objects, the host only needs to distribute the marker information (e.g., ArUco marker), rather than keyframes and features as in SLAMbased AR. The marker information can be compactly represented as an image or ID number, and thus is communication-efficient in which case SPAR is not needed.

Cloud vs. P2P architectures: SPAR currently uses a P2P architecture to distribute AR information directly to each resolving client, as do Apple ARKit and Microsoft Hololens. A P2P architecture is a natural fit for AR, since AR information only needs to be distributed in a geographically restricted area. However, SPAR could be modified to run coordinate system synchronization on a central node, such as a cloud or edge server (for example, Google ARCore 
uses the cloud), although privacy is a concern. In this case, communication latency may increase, but computation latency may decrease, requiring further evaluation of the tradeoffs.

\section{Related Work}

Mobile AR systems: Object detection and image recognition for AR, on device or offloaded to the edge/cloud, has been investigated $[7,12,22,26,32-34,42,49]$ in order to place virtual objects in the real world. These works are orthogonal to ours as we assume that the virtual objects' locations is given (by object detection or user input), and we focus on how AR users can coordinate this information with others. VisualPrint [27] uses visual fingerprints for localization, whereas we use SLAM for localization as common in commercial AR platforms. While MARVEL [11] studies 6-DoF based AR systems, they assume the real world is pre-mapped, whereas we assume that devices are placed in an unknown environment. EdgeSLAM [5] considers offloading parts of SLAM to an edge server, whereas SPAR does not require infrastructure support. GLEAM [40] focuses on lighting rendering for virtual objects, which is complementary to this work. Recent work [48] proposes geo-visual techniques for fast localization in urban areas; however, their AR system is single-user whereas we focus on multi-user scenarios.

Multi-user AR: CARS [50] shares results from object detection among multiple users, whereas this paper focuses on more general 3D coordinate system alignment to share virtual objects including those placed by object detection. CarMap [4] proposes efficient map compression, without any virtual objects; in contrast, SPAR uses knowledge of the virtual object positions when deciding what to communicate. Several works $[6,43]$ present only preliminary measurements of multi-user AR. While industry multi-user AR systems such as Google ARCore [20], Apple ARKit [8], and Microsoft HoloLens [35] are close-sourced, we study communication and spacial inconsistency aspects of multi-user AR through an open-source system [31]

Multi-agent SLAM: Some SLAM systems [3, 24] focus on coordinate system alignment, while other work [14, 25] assumes advanced sensors such as 2D laser scanner or 3D LiDARs. In contrast, this paper focuses on efficient SLAM-based communications on commodity smartphones, which have a large potential user base. Zou et al. [53] hardcodes transmitting the SLAM data up to every 5 frames, while CCM-SLAM [46] transmits SLAM information whenever it is updated. Instead, we select the appropriate keyframes and their associated point clouds based on the locations of the virtual objects. This is done on top of the default keyframe selection already performed by SLAM frameworks such as ORB-SLAM2 [38] or VINS [41].

In terms of frameworks, we work with VINS-AR [31], which is an Android version of VINS-Mono [41], both of which are single-user SLAM and do not consider communication and consistency issues of multi-user AR. Other open-source SLAM systems are either not tested on Android [16, 46] or do not utilize IMU sensors [38].

\section{Conclusions}

In this paper, we investigated communication and computation bottlenecks of multi-user AR applications. We found that off-theshelf AR apps suffer from high communication latency and inconsistent placement of the virtual objects across users and across time.
We proposed solutions for efficient data communications between AR users to reduce latency while maintaining accurate positioning of the virtual objects, as well as a quantitative method of estimating these positioning changes.

Our implementation on an open-source Android AR platform demonstrated the efficacy of the proposed solutions. Future work includes extending our spatial inconsistency tool to other AR platforms such as ARCore, as well as incorporating depth cameras.

\section{Acknowledgements}

This paper benefited significantly from feedback from the CoNEXT 2020 reviewers and shepherd, for which we are very grateful. This work was supported in part by NSF CAREER 1942700, CSR-1903136, and CNS-1908051.

\section{References}

[1] Boost c libraries. https://www.boost.org/

[2] Abawi, D. F., Bienwald, J., AND Dorner, R. Accuracy in optical tracking with fiducial markers: an accuracy function for artoolkit. In IEEE ISMAR (Nov 2004).

[3] Abdulgalil, M. A., Nasr, M. M., Elalfy, M. H., Khamis, A., and Karray, F. Multirobot slam: An overview and quantitative evaluation of mrgs ros framework for mr-slam. In International Conference on Robot Intelligence Technology and Applications (2017), Springer, pp. 165-183.

[4] Ahmad, F., Qiu, H., Eells, R., Bai, F., And Govindan, R. Carmap: Fast 3d feature map updates for automobiles. In USENIX NSDI (2020), pp. 1063-1081.

[5] Ali, A. J. B., Hashemifar, Z. S., And Dantu, K. Edge-slam: edge-assisted visual simultaneous localization and mapping. In ACM MobiSys (2020), pp. 325-337.

[6] Apicharttrisorn, K., Balasubramanian, B., Chen, J., Sivaraj, R., Tsai, Y.Z., Jana, R., Krishnamurthy, S., Tran, T., ANd Zhou, Y. Characterization of multi-user augmented reality over cellular networks. In IEEE SECON (2020).

[7] Apicharttrisorn, K., Ran, X., Chen, J., Krishnamurthy, S., and RoyChowdhury, A. Frugal following: Power thrifty object detection and tracking for mobile augmented reality. ACM SenSys (2019).

[8] Apple. Creating a multiuser ar experience. https:/developer.apple.com/ documentation/arkit/creating_a_multiuser_ar_experience.

[9] Bradski, G. The OpenCV Library. Dr. Dobb's Journal of Software Tools (2000).

[10] Bradski, G., AND Kaehler, A. Learning OpenCV: Computer vision with the OpenCV library. " O’Reilly Media, Inc.", 2008.

[11] Chen, K., Li, T., Kim, H.-S., Culler, D. E., And Katz, R. H. MARVEL: Enabling mobile augmented reality with low energy and low latency. ACM Sensys (2018).

[12] Chen, T. Y.-H., Ravindranath, L., Deng, S., Bahl, P., and Balakrishnan, H. Glimpse: Continuous, real-time object recognition on mobile devices. ACM SenSys (2015).

[13] Chen, Z., Hu, W., Wang, J., Zhao, S., Amos, B., Wu, G., Ha, K., Elgazzar, K., Pillai, P., Klatzky, R., ET AL. An empirical study of latency in an emerging class of edge computing applications for wearable cognitive assistance. In Proceedings of the Second ACM/IEEE Symposium on Edge Computing (2017), pp. 1-14.

[14] Dubé, R., Gawel, A., Sommer, H., Nieto, J., Siegwart, R., and Cadena, C. An online multi-robot slam system for $3 \mathrm{~d}$ lidars. In IEEE/RSf International Conference on Intelligent Robots and Systems (Sep. 2017), pp. 1004-1011.

[15] Durrant-Whyte, H., And Bailey, T. Simultaneous localization and mapping: part i. IEEE Robotics Automation Magazine 13, 2 (2006), 99-110.

[16] Forster, C., Zhang, Z., Gassner, M., Werlberger, M., and Scaramuzza, D. Svo: Semidirect visual odometry for monocular and multicamera systems. IEEE Transactions on Robotics 33, 2 (2016), 249-265.

[17] Gálvez-López, D., AND TaRdós, J. D. Bags of binary words for fast place recognition in image sequences. IEEE Transactions on Robotics 28, 5 (October 2012), $1188-1197$.

[18] Garrido-Jurado, S., Muñoz-Salinas, R., Madrid-Cuevas, F. J., and MarínJimÉnez, M. J. Automatic generation and detection of highly reliable fiducial markers under occlusion. Pattern Recognition 47, 6 (2014), 2280-2292.

[19] Google. Arcore overview. https://developers.google.com/ar/discover/.

[20] Google. Share ar experiences with cloud anchors. https://developers.google.com/ ar/develop/java/cloud-anchors/cloud-anchors-overview-android, May 2018.

[21] Google Creative Labs. Just a Line - Draw Anywhere, with AR. https://justaline. withgoogle.com/

[22] Ha, K., Chen, Z., Hu, W., Richter, W., Pillai, P., and Satyanarayanan, M. Towards wearable cognitive assistance. ACM MobiSys (2014).

[23] Holloway, R. L. Registration errors in augmented reality systems. PhD thesis, Citeseer, 1995.

[24] Howard, A. Multi-robot simultaneous localization and mapping using particle filters. I. F. Robotic Res. 25 (12 2006), 1243-1256. 
[25] JAFRI, S., AND Chellali, R. A distributed multi robot slam system for environment learning. In IEEE Workshop on Robotic Intelligence in Informationally Structured Space (2013).

[26] Jain, P., Manweiler, J., And Roy Choudhury, R. Overlay: Practical mobile augmented reality. ACM MobiSys (2015).

[27] Jain, P., MANweiler, J., AND Roy Choudhury, R. Low bandwidth offload for mobile AR. ACM CoNEXT (2016)

[28] Jinyu, L., Bangbang, Y., Danpeng, C., Nan, W., Guofeng, Z., and Hujun, B. Survey and evaluation of monocular visual-inertial slam algorithms for augmented reality. Virtual Reality \& Intelligent Hardware 1, 4 (2019), 386-410.

[29] Kyoung Shin Park, And Kenyon, R. V. Effects of network characteristics on human performance in a collaborative virtual environment. In IEEE Virtual Reality (Mar. 1999).

[30] LaValle, S. Virtual Reality. Cambridge University Press.

[31] Li, P., Qin, T., Hu, B., Zhu, F., And Shen, S. Monocular visual-inertial state estimation for mobile augmented reality. In IEEE ISMAR (2017), pp. 11-21.

[32] Liu, L., Li, H., AND Gruteser, M. Edge assisted real-time object detection for mobile augmented reality. ACM MobiCom (2019).

[33] Liu, Q., AND HAN, T. Dare: Dynamic adaptive mobile augmented reality with edge computing. IEEE ICNP (2018).

[34] Liu, Z., Lan, G., Stojkovic, J., Zhang, Y., Joe-Wong, C., And Gorlatova, M Collabar: Edge-assisted collaborative image recognition for mobile augmented reality. In ACM/IEEE IPSN (2020)

[35] Microsoft. Shared experiences in unity. https://docs.microsoft.com/en-us/ windows/mixed-reality/shared-experiences-in-unity, March 2018

[36] Moeller, J. jannismoeller/vins-mobile-android. https:/github.com/ jannismoeller/VINS-Mobile-Android, Jul 2019.

[37] Mojang. Minecraft for Android. https://www.minecraft.net/en-us/store/ minecraft-android/.

[38] Mur-Artal, R., AND TARDós, J. D. Orb-slam2: An open-source slam system for monocular, stereo, and rgb-d cameras. IEEE Transactions on Robotics 33, 5 (2017), $1255-1262$.

[39] Niantic. Pokemon Go. https://www.pokemongo.com/en-us/.

[40] Prakash, S., Bahremand, A., Nguyen, L. D., and LiKamWa, R. GLEAM: An Illumination Estimation Framework for Real-time Photorealistic Augmented
Reality on Mobile Devices. ACM MobiSys (2019).

[41] Qin, T., Li, P., AND Shen, S. Vins-mono: A robust and versatile monocular visual-inertial state estimator. IEEE Transactions on Robotics 34, 4 (Aug 2018), 1004-1020.

[42] Ran, X., Chen, H., Liu, Z., And Chen, J. Deepdecision: A mobile deep learning framework for edge video analytics. IEEE INFOCOM (2018).

[43] Ran, X., Slocum, C., Gorlatova, M., and Chen, J. Sharear: Communicationefficient multi-user mobile augmented reality. In ACM HotNets Workshop (2019), pp. 109-116.

[44] Romero-Ramirez, F., Muñoz-Salinas, R., and Medina-Carnicer, R. Speeded up detection of squared fiducial markers. Image and Vision Computing 76 (06 2018).

[45] Schmalstieg, D., And Hollerer, T. Augmented reality: principles and practice. Addison-Wesley Professional, 2016.

[46] Schmuck, P., AND CHLI, M. Multi-uav collaborative monocular slam. IEEE International Conference on Robotics and Automation (2017).

[47] Sturm, J., Engelhard, N., Endres, F., Burgard, W., And Cremers, D. A benchmark for the evaluation of rgb-d slam systems. In IEEE/RSF International Conference on Intelligent Robots and Systems (2012).

[48] Xu, T., Wang, G., AND Lin, F. X. Practical urban localization for mobile ar. In ACM HotMobile Workshop (2020).

[49] Zhang, W., Han, B., ANd Hui, P. Jaguar: Low Latency Mobile Augmented Reality with Flexible Tracking. ACM Multimedia (2018).

[50] Zhang, W., Han, B., Hui, P., Gopalakrishnan, V., Zavesky, E., and Qian, F. Cars: Collaborative augmented reality for socialization. ACM HotMobile (2018).

[51] Zheng, F. Spatio-temporal registration in augmented reality. $\mathrm{PhD}$ thesis, The University of North Carolina at Chapel Hill, 2015.

[52] Zheng, Y., Kunng, Y., Sugimoto, S., Åström, K., And Oкutomi, M. Revisiting the pnp problem: A fast, general and optimal solution. In IEEE ICCV (2013), pp. 2344-2351.

[53] Zou, D., AND TAN, P. Coslam: Collaborative visual slam in dynamic environments. IEEE Transactions on Pattern Analysis and Machine Intelligence 35, 2 (2012), 354366. 